\title{
Traumatismo torácico en el adulto mayor
}

\author{
Roberto González L. ${ }^{1,2}$, Alberto Fuentes E. ${ }^{3}$, Alejandra Riquelme U. ${ }^{4}$, \\ Rodrigo Reyes M. ${ }^{1,2}$, Enrique Seguel S. ${ }^{1,2}$, Aleck Stockins L. ${ }^{1,2}$, \\ Andrés Jadue T. ${ }^{1}$, Manuel Rivera J. ${ }^{3}$ y Emilio Alarcón C. ${ }^{1,2}$
}

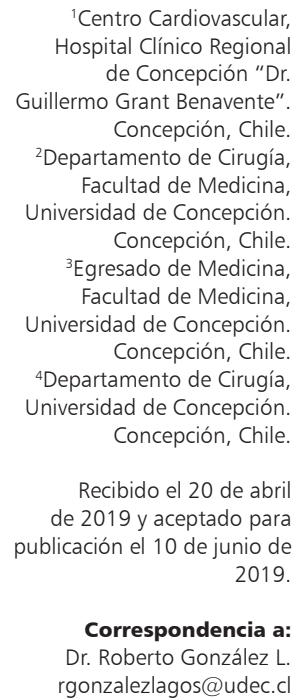

Dr. Roberto González L.

\section{Thoracic trauma in the elderly}

Introduction: Trauma is the fifth leading cause of death in the elderly (60 or older), with blunt trauma being the most frequent. Objective: To describe characteristics, Trauma Severity Indices (TSI) and morbidity and mortality in hospitalized elderly for Thoracic Trauma (TT). Materials and Method: Crosssectional descriptive study was carried out. Term: from January 1981 to December 2017. Database review, surgical protocols and medical records were performed. TT description of characteristics was conducted in hospitalized elderly. TSI was calculated: Injury Severity Score (ISS), Revised Trauma Score (RTS-T), Trauma Injury Severity Score (TRISS). Results: Total 4.163 TT, 513 elderly (12.3\%). Men: 350 (68.2\%), average age $71.2 \pm 8.4$ years, 70 median (range 60-103). Isolated TT: $350(68.2 \%), 163$ associated with extrathoracic trauma $(31.8 \%)$ and of these $96(18.7 \%)$ were considered polytraumatism. Blunt trauma $456(88.9 \%)$ and penetrating 57 (11.1\%). Most frequent domestic accident mechanism was $196(38.2 \%)$ and 158 traffic accidents (30.8\%). Frequently thoracic injuries or findings: 409 rib fractures (79.7\%), 186 hemothorax (36.3\%), and 185 pneumothorax (36.1\%). Final treatment: 287 Medical treatment $(55.9 \%)$, 193 pleurotomy $(37.6 \%)$, and 40 thoracic surgery $(7.8 \%)$. Extrathoracic surgery $33(6.4 \%)$. Average hospitalization: $9.0 \pm 8.8$ days. According IGT: ISS $12.1 \pm 9.6$, RTS-T $11.6 \pm 1.3$, TRISS 8.1. Morbidity: 76 (14.8\%) and mortality: 26 (5.1\%). Discussion: Most TT in elderly are blunt, caused by domestic accidents. Injuries and most frequent findings were rib fractures and hemothorax. Mortality was lower than expected according to TSI.

Key words: elderly; thoracic trauma; trauma severity indices; thoracic surgery; geriatric trauma.

\section{Resumen}

Introducción: Los traumatismos constituyen la quinta causa de muerte en el adulto mayor (60 años o más), siendo los traumatismos contusos los más frecuentes. Objetivo: Describir características, índices de gravedad de traumatismo (IGT), morbilidad y mortalidad en adultos mayores (AM) hospitalizados con traumatismo torácico (TT). Materiales y Método: Estudio descriptivo transversal. Período desde enero de 1981 a diciembre de 2017. Revisión de base de datos, protocolos quirúrgicos y fichas clínicas. Descripción de características de TT en AM hospitalizados. Se calculó IGT: Injury Severity Score (ISS), Revised Trauma Score (RTS-T), Trauma Injury Severity Score (TRISS). Resultados: Total 4.163 TT, AM 513 (12,3\%). Hombres: 350 (68,2\%), edad promedio 71,2 $\pm 8,4$ años, mediana 70 (rango: 60-103), TT aislado $350(68,2 \%)$, asociado a lesiones extratorácicas $163(31,8 \%)$ y de estos $96(18,7 \%)$ se consideraron politraumatismos. Traumatismo contuso $456(88,9 \%)$ y penetrante $57(11,1 \%)$. La causa más frecuente fueron las caídas en $252(49,1 \%)$. Lesiones y/o hallazgos torácicos más frecuentes: fracturas costales 409 (79,7\%), hemotórax $186(36,3 \%)$ y neumotórax $185(36,1 \%)$. Tratamiento definitivo: médico $287(55,9 \%)$, pleurotomía 193 (37,6\%) y cirugía 40 (7,8\%). Cirugía extratorácica $33(6,4 \%)$. Hospitalización promedio $9,0 \pm 8,8$ días. Según IGT: ISS promedio $12,1 \pm 9,6$, RTS-T promedio $11,6 \pm 1,3$, TRISS promedio 8,1 . Morbilidad 76 (14,8\%) y mortalidad 26 (5,1\%). Discusión: La mayoría de los TT en AM son contusos, causados por accidentes domésticos. Las lesiones y hallazgos más frecuentes fueron fracturas costales y hemotórax. La mortalidad fue menor a la esperada según IGT.

Palabras clave: ancianos; trauma torácico; índices de gravedad del traumatismo; cirugía torácica; traumatismo geriátrico. 


\section{Introducción}

Según la Organización Mundial de la Salud (OMS) y el Instituto Nacional de Estadísticas de Chile (INE), se define como adulto mayor (AM) a toda persona mayor o igual a 60 años, tercera edad entre 60 y 79 años y cuarta edad a toda persona mayor o igual de 80 años $^{1,2}$. En esta población, los traumatismos adquieren especial importancia debido a la fragilidad inherente tanto constitucional como funcional $^{3-5}$.

Se estima que los ingresos a urgencia de AM traumatizados corresponden a un tercio de las hospitalizaciones por traumatismo ${ }^{6,7}$. Existe poca información en la literatura mundial acerca del traumatismo torácico del adulto mayor (TTAM), a pesar que, los traumatismos constituyen la quinta causa de muerte en este grupo etario ${ }^{8}$.

Nuestros objetivos son describir características, índices de gravedad de traumatismo (IGT), morbilidad y mortalidad en AM hospitalizados con TT en nuestro hospital.

\section{Materiales y Método}

Se realizó estudio descriptivo transversal de los pacientes adultos mayores hospitalizados con traumatismo torácico (TT) ingresados al Hospital Clínico Regional "Dr. Guillermo Grant Benavente" de Concepción-Chile, tratados por el Equipo de Cirugía Cardiotorácica, en el periodo comprendido entre enero de 1981 y diciembre de 2017. Se utilizaron las definiciones de AM según las recomendaciones de la OMS y del INE de Chile ${ }^{1,2}$.

La información se obtuvo a partir de una base de datos prospectiva de traumatismo torácico, fichas clínicas y protocolos quirúrgicos. Se tabularon y analizaron los datos mediante planilla Microsoft Excel $^{\circledR}$ y programa SPSS $21^{\circledR}$ con función $t d e S t u$ dent para variables cuantitativas y $\chi^{2}$ para variables cualitativas.

Se describen edad, sexo, características clínicas, mecanismo, tipo de traumatismo, morbilidad, mortalidad y estadía hospitalaria. Se calcularon índices de gravedad de traumatismo (IGT): ISS (Injury Severity Score), RTS-T (Revised Trauma Score Triage) y TRISS (Trauma Injurity Severity Score). Se compararon características y resultados entre la tercera edad y cuarta edad.

Respecto de los scores de traumatismo utilizados, ISS determina la gravedad de acuerdo a lesión anatómica ${ }^{9}$, RTS-T de acuerdo al estado o deterioro fisiológico al momento del ingreso (presión arterial sistólica, frecuencia respiratoria y escala de Glas- gow $)^{10}$ y TRISS, que corresponde a un índice mixto (anatómico-fisiológico), determina el riesgo de mortalidad considerando, además, la edad del paciente y el mecanismo del traumatismo ${ }^{11,12}$. Se definió como politraumatizado a los que presentaron un ISS $>16$ puntos ${ }^{13,14}$.

El estudio y análisis de las hospitalizaciones por traumatismo torácico fue autorizado por el comité de ética de nuestra institución.

\section{Resultados}

El total de TT hospitalizados fue de 4.163 pacientes, de ellos $513(12,3 \%)$ fueron adultos mayores (AM), de los cuales $419(81,7 \%)$ fueron de la tercera edad y $94(18,3 \%)$ de la cuarta edad. Hombres 350 $(68,2 \%)$ y $163(31,8 \%)$ mujeres, con una relación Hombre: Mujer de 2,15: 1. La edad promedio fue de $71,2 \pm 8,4$ años, con mediana de 70 y un rango entre 60 y 103 años.

Fueron traumatismos contusos $456(88,9 \%)$ y penetrantes $57(11,1 \%)$. La mayor parte de los TT fueron aislados y el mecanismo traumático más frecuente fue el accidente doméstico en $196(38,2 \%)$, seguido de accidente de tránsito en $158(30,8 \%)$, de estos $69(44 \%)$ fueron atropellos (Tabla 1). Cerca de

Tabla 1. Sexo, edad, clasificación, tipo y causa del traumatismo torácico en adulto mayor

\begin{tabular}{|ll|}
\hline Total & $\mathbf{5 1 3}$ \\
\hline Sexo & \\
Hombre & $350(68,2 \%)$ \\
Mujer & $163(31,8 \%)$ \\
Edad & \\
Promedio & $71,2 \pm 8,4$ \\
Mediana & 70 \\
Rango & $60-103$ \\
Tercera edad & $419(81,7 \%)$ \\
Cuarta edad & $94(18,3 \%)$ \\
Clasificación & \\
Contuso & $456(88,9 \%)$ \\
Penetrante & $57(11,1 \%)$ \\
Tipo de traumatismo & \\
TT aislado & $350(68,2 \%)$ \\
TT con lesiones asociadas & $163(31,8 \%)$ \\
Politraumatismo & $96(18,7 \%)$ \\
Causa & \\
Accidente doméstico & $196(38,2 \%)$ \\
Accidente de tránsito & $158(30,8 \%)$ \\
Agresión & $49(9,6 \%)$ \\
Accidente laboral & $21(4,1 \%)$ \\
Otra causa & $89(17,3 \%)$ \\
\hline
\end{tabular}

TT: Traumatismo torácico. 
Tabla 2. Sitio, agentes o mecanismo más frecuentes del traumatismo torácico en adulto mayor

\begin{tabular}{lr}
\hline Total & $\mathbf{5 1 3}$ \\
Sitio de traumatismo & \\
Vía pública & $278(54,2 \%)$ \\
Domicilio & $196(38,2 \%)$ \\
Otros & $39(7,6 \%)$ \\
Agente o mecanismo & \\
Caídas & $252(49,1 \%)$ \\
Vehículo motorizado & $153(29,8 \%)$ \\
Objeto contundente & $56(10,9 \%)$ \\
Arma blanca & $41 \quad(8,0 \%)$ \\
Arma de fuego & $6(1,1 \%)$ \\
Vehículo no motorizado & $5(1,0 \%)$ \\
\hline
\end{tabular}

la mitad de todos los TT $(49,1 \%)$ fueron causados por caídas (Tabla 2 ).

Las lesiones y/o hallazgos torácicos más frecuentes fueron las fracturas costales en 409 $(79,7 \%)$ y el hemotórax en $186(36,3 \%)$ lo que se observa en las Tablas 3 y 4 . Las lesiones extratorácicas asociadas más frecuentes fueron el traumatismo encefalocraneano en $82(16,0 \%)$ y las fracturas de extremidades en $59(11,5 \%)$, lo que se observa en la Tabla 5.

En relación al tratamiento definitivo, requirieron tratamiento médico $287(55,9 \%)$ pacientes y 226 $(44,1 \%)$ se resolvieron con tratamiento invasivo (Tabla 6). En 33 (6,4\%) pacientes se realizó cirugía extratorácica: $15(2,9 \%)$ abdominales, $11(2,1 \%)$ traumatológicas, $5(1,0 \%)$ de partes blandas y 2 $(0,4 \%)$ vasculares. Hubo $2(0,4 \%)$ reoperaciones torácicas y $2(0,4 \%)$ extratorácicas. Tiempo de hospitalización, morbilidad, mortalidad e IGT se detallan en la Tabla 7.

Al comparar la tercera y cuarta edad, se observó en la tercera edad significativamente una mayor proporción de politraumatismos, traumatismos por accidentes de tránsito y agresiones, además, mayor morbilidad, estadía hospitalaria y gravedad anatómica (ISS), sin diferencias significativas en mortalidad, TRISS ni RTS-T (Tabla 8). En el grupo específico de pacientes politraumatizados la mortalidad fue de $14 \%$ (12/86 pacientes) en la tercera edad y de $30 \%$ (3/10 pacientes) en la cuarta edad.
Tabla 3. Hallazgos y/o lesiones torácicas en el traumatismo torácico en adulto mayor

\begin{tabular}{|lr|}
\hline Total & $\mathbf{5 1 3}$ \\
Fracturas costales & $409(79,7 \%)$ \\
Hemotórax & $186(36,3 \%)$ \\
Neumotórax & $185(36,1 \%)$ \\
Enfisema subcutáneo & $136(26,5 \%)$ \\
Contusión pulmonar & $100(19,5 \%)$ \\
Fractura esternal & $42(8,2 \%)$ \\
Penetrante cardíaca & $12(2,3 \%)$ \\
Lesión diafragmática & $9(1,8 \%)$ \\
Lesión parénquima pulmonar & $5(1,0 \%)$ \\
Lesión de grandes vasos & $5(1,0 \%)$ \\
Neumotórax abierto & $1 \quad(0,2 \%)$ \\
\hline
\end{tabular}

Tabla 4. Fracturas costales y sus complicaciones en el traumatismo torácico en adulto mayor

\begin{tabular}{|lc|}
\hline Total & $\mathbf{5 1 3}$ \\
\hline 1 a 3 costillas & $204(39,8 \%)$ \\
4 a 6 costillas & $144(28,1 \%)$ \\
\hline 6 costillas & $61(11,9 \%)$ \\
Tórax volante & $50(9,8 \%)$ \\
\hline Fractura costal complicada & $259(50,5 \%)$ \\
\hline Complicación intratorácicas & $255(49,7 \%)$ \\
Complicación extratorácicas & $24(4,7 \%)$ \\
\hline
\end{tabular}

Tabla 5. Lesiones extratorácicas en el traumatismo torácico en adulto mayor

\begin{tabular}{|c|c|}
\hline Total & 513 \\
\hline Craneoencefálicas & $82(16,0 \%)$ \\
\hline Extremidades & $59(11,5 \%)$ \\
\hline Abdominales & $35(6,8 \%)$ \\
\hline Fracturas de pelvis & $25(4,9 \%)$ \\
\hline Traumatismo facial & $23(4,5 \%)$ \\
\hline Lesión de partes blandas & $21 \quad(4,1 \%)$ \\
\hline
\end{tabular}

Tabla 6. Tratamiento definitivo en el traumatismo torácico en adulto mayor

\begin{tabular}{|ll|}
\hline Total & $\mathbf{5 1 3}$ \\
\hline Tratamiento médico exclusivo & $287(55,9 \%)$ \\
\hline Tratamiento invasivo & $226(44,1 \%)$ \\
Pleurotomía exclusiva & $186(36,3 \%)$ \\
Cirugía & $40(7,8 \%)$ \\
\hline
\end{tabular}


Tabla 7. Morbilidad, mortalidad, estadía hospitalaria e IGT en el traumatismo torácico en adulto mayor

\begin{tabular}{|lc|}
\hline Total & $\mathbf{5 1 3}$ \\
Morbilidad & $\mathbf{7 6}(\mathbf{1 4 , 8 \% )}$ \\
Atribuida a TT & $73(14,2 \%)$ \\
Atribuida a cirugía torácica & $3(0,6 \%)$ \\
Mortalidad & $\mathbf{2 6} \mathbf{( 5 , 1 \% )}$ \\
0 - 7 días desde TT & $13(2,5 \%)$ \\
8 - 30 días desde TT & $9(1,8 \%)$ \\
$>30$ días desde TT & $4(0,8 \%)$ \\
Estadía hospitalaria & \\
Promedio en días & $9,0 \pm 8,8$ \\
Mediana & 6 \\
Rango & $1-83$ \\
Índices de gravedad del traumatismo & Promedio \\
Injury Severity Score (ISS) & $12,1 \pm 9,6$ \\
Revised Trauma Score Triage (RTS-T) & $11,6 \pm 1,3$ \\
Trauma Injury Severity Score (TRISS) (\% mortalidad esperada) & 8,1 \\
Mortalidad observada & $5,1 \%$ \\
\hline
\end{tabular}

TT: Traumatismo torácico.

\section{Discusión}

En un trabajo recientemente publicado por nuestro grupo, revisamos la evolución de las hospitalizaciones por TT durante tres décadas ${ }^{7}$. En este periodo observamos un aumento significativo de las hospitalizaciones por TT en la población mayor de 65 años, lo que nos motivó a realizar un estudio dirigido a caracterizar los adultos mayores.

El AM es especialmente susceptible a los traumatismos. Esto debido a diversos factores asociados al proceso de envejecimiento, tales como la disminución de la agudeza visual y auditiva, reflejos disminuidos, movilidad limitada y sarcopenia, entre otros $^{3-6,15-18}$. Asimismo, debido al deterioro anatomofisiológico que la senilidad conlleva, la respuesta del organismo frente a un traumatismo se encuentra modificada, por lo que presentan mayor morbilidad y mortalidad asociada al traumatismo ${ }^{17,18}$. La gravedad, mal pronóstico y mortalidad del AM con TT dependen de muchos componentes que deben ser considerados. En primer lugar hay factores inherentes al tipo de traumatismo (penetrante o contuso), siendo el contuso el que adquiere mayor importancia

Tabla 8. Tipo, clasificación, causa, morbilidad, mortalidad e IGT en el traumatismo torácico entre tercera y cuarta edad

\begin{tabular}{|c|c|c|c|c|}
\hline & Total (513) & Tercera edad (419) & Cuarta edad (94) & $\mathbf{p}$ \\
\hline Aislado & $350(68,2 \%)$ & $274(65,4 \%)$ & $76(80,9 \%)$ & $0,004^{*}$ \\
\hline Politraumatismo & $96(18,7 \%)$ & $86(20,5 \%)$ & $10(10,6 \%)$ & $0,02 *$ \\
\hline \multicolumn{5}{|l|}{ Clasificación } \\
\hline \multicolumn{5}{|l|}{ Causas principales } \\
\hline Accidente doméstico & $196(38,2 \%)$ & $134(32,0 \%)$ & $62(66,0 \%)$ & $0,001 *$ \\
\hline Accidente de tránsito & $158(30,8 \%)$ & $141(33,7 \%)$ & $17(18,1 \%)$ & $0,003 *$ \\
\hline Agresión & $49 \quad(9,6 \%)$ & $47(11,2 \%)$ & $2(2,1 \%)$ & $0,007 *$ \\
\hline Morbilidad & $76(14,8 \%)$ & $69(16,5 \%)$ & $7(7,5 \%)$ & $0,02 *$ \\
\hline \multicolumn{5}{|l|}{ Índices de gravedad del traumatismo (promedio) } \\
\hline Injury Severity Score (ISS) & $2,1 \pm 9,6$ & $12,7 \pm 9,7$ & $9,4 \pm 8,6$ & $0,002 *$ \\
\hline Revised Trauma Score Triage (RTS-T) & $11,6 \pm 1,3$ & $11,6 \pm 1,3$ & $11,8 \pm 1,1$ & 0,284 \\
\hline Trauma Injury Severity Score (TRISS) (\% mortalidad esperada) & $8,1 \%$ & $8,6 \%$ & $6,0 \%$ & 0,577 \\
\hline
\end{tabular}

*Estadísticamente significativo; TT: Traumatismo torácico. **En el grupo específico de pacientes politraumatizados la mortalidad fue más del doble en pacientes de la cuarta edad con respecto a la tercera edad. 
en el $\mathrm{AM}^{11,12}$. También es importante indagar sobre el mecanismo que provocó el TT y no subestimarlo, pues una simple caída a nivel que pudiese ser inofensiva en un paciente joven, en el AM puede conllevar complicaciones significativas. La biomecánica e interpretación de las lesiones, según la intensidad, energía, ángulo, agente traumático, dirección del agente traumático, mecanismo y sitio de lesión son aspectos fundamentales al momento de enfrentarse al paciente, pues revelan información esencial sobre las posibles estructuras torácicas y extratorácicas afectadas $^{14}$. En cuanto a los factores del AM se encuentran: el deterioro orgánico asociado al proceso mismo de envejecimiento, la desmineralización ósea, las deficiencias sensoriales, menor musculatura torácica y enfermedades crónicas, entre otras ${ }^{4-6}$. Además, el alcoholismo y polifarmacia son factores adquiridos que se relacionan con mal pronóstico ${ }^{8}$.

Los traumatismos constituyen la quinta causa de muerte en el AM, siendo solo superado por las enfermedades cardiacas, cáncer, neumonía y complicaciones de la diabetes mellitus ${ }^{6}$. Hay que tener en cuenta que más del $60 \%$ de los pacientes ancianos traumatizados que ingresan inconscientes a un servicio de urgencias no sobreviven a pesar de una resucitación adecuada ${ }^{6}$. Además, se sabe que pacientes mayores de 85 años tienen aproximadamente 4 veces más probabilidades de muerte que los de 70-75 años frente a un traumatismo ${ }^{6}$.

Actualmente, se estima que un tercio de los pacientes hospitalizados por traumatismos son adultos mayores ${ }^{18}$. Esto se explica en parte por el sorprendente aumento de la población geriátrica a nivel mundial y por una mayor flexibilidad al decidir su hospitalización. En nuestro país durante la mayor parte del siglo XX, los AM representaban solo el $1 \%$ de la población, sin embargo, para el año 2020 se espera un aumento cercano al $20 \% 0^{5}$, esto genera la necesidad apremiante de formar personal médico apto para enfrentar este tipo de pacientes.

En nuestro país, el mecanismo más frecuente de TT en personas jóvenes es la agresión ${ }^{7}$. Sin embargo, en el AM este mecanismo pasa a un tercer plano dejando en primer lugar las caídas y en segundo lugar los accidentes de tránsito ${ }^{6,10-12}$, destacando que en nuestra serie casi la mitad de los accidentes de tránsito son atropellos. Si bien en algunas publicaciones la frecuencia cambia, en nuestra serie cerca de la mitad de los TT fueron a causa de caídas. Bajo la misma línea, la gran mayoría de los TTAM son causados por objetos contundentes, al contrario de lo que ocurre en nuestra población joven, donde el traumatismo penetrante es el que predomina ${ }^{7,15,19-22}$. Nuestros datos muestran que la mayoría de los AM presentaron TT contusos y domésticos, principalmente en aquellos de la cuarta edad. Por esto, cada vez cobra más valor la utilización de medidas de salud no solo centradas en las morbilidades, factores de riesgo y enfermedades crónicas que estos padecen, sino también en el bienestar, calidad de envejecimiento y entorno físico en el cual se desenvuelven ${ }^{3}$.

Según distintas series, los traumatismos torácicos en la población general son asociados a lesiones extratorácicas hasta en un $70 \%$ de los casos, principalmente injurias a nivel craneoencefálico, abdominal y traumatológico, lo que aumenta su complejidad $^{15,19-23}$. En nuestra serie solo un tercio de los pacientes tuvo lesiones asociadas.

En cuanto a las lesiones torácicas más frecuentemente descritas en la literatura, las fracturas costales ocupan el primer lugar (50\%), seguidas de las contusiones pulmonares, hemotórax y neumotórax ${ }^{15,19-22}$. En nuestra serie las principales lesiones torácicas asociadas fueron las fracturas costales, seguido del hemotórax y neumotórax.

La mortalidad asociada a traumatismo varía entre $2,2 \%-15 \%$ según las distintas series ${ }^{7,15,19-22}$, la que aumenta hasta un $19,5 \%$ en el adulto mayor. Según la literatura, en el sitio del accidente las causas de muerte son principalmente obstrucción de la vía aérea, lesiones cardiacas $^{24}$ y lesiones de grandes vasos; durante las primeras horas de evolución son neumotórax a tensión, contusiones pulmonares y hemorragias; en los días siguientes al traumatismo corresponden a complicaciones como sepsis y falla multiorgánica ${ }^{25,26}$. En nuestro caso la mortalidad se presentó con mayor frecuencia durante la primera semana postraumatismo, siendo globalmente un $5,1 \%$, menor a la esperada según TRISS $(8,1 \%)$.

Al comparar la tercera y cuarta edad, se observó una mayor morbilidad y estadía hospitalaria en el primer grupo, esto asociado a un mayor porcentaje de accidentes de tránsito y politraumatismos reflejados en el aumento del ISS. No se observó diferencias significativas en la mortalidad global a pesar del mayor valor de ISS en la tercera edad, lo que se podría explicar dado a que presentan una menor proporción de traumatismos contusos y menor edad, lo que influencia directamente el valor del TRISS y la mortalidad, por lo que a pesar de ser pacientes más graves anatómicamente, tienen una mortalidad similar que los de la cuarta edad que son más seniles y con más traumatismos contusos.

Por otro lado, cabe descatar que en el grupo específico de pacientes politraumatizados, la mortalidad fue más del doble en los adultos mayores de la cuarta edad respecto a los de la tercera edad. 
En este subgrupo el impacto de la fragilidad y comorbilidades cobra mayor importancia, elevando considerablemente la mortalidad.

Podemos decir que existe poca información sobre TTAM en la literatura, siendo de vital importancia conocer sus características propias, dada la fragilidad orgánica que estos presentan. Por otra parte, la expectativa demográfica ha ido variando considerablemente, razón por la cual cada vez hay mayor proporción de AM. En nuestra serie casi el $80 \%$ de los pacientes presentaron fracturas costales y la mitad de éstos tuvo complicaciones (en su mayoría torácicas), lo cual es de suma importancia ya que las características propias del AM, los llevan a presentar más complicaciones y peor pronóstico si se subestiman y no pesquisan o tratan oportunamente sus lesiones, principalmente, en el caso de los politraumatizados.

A modo de conclusión, el TTAM tiene características particulares que lo diferencian de la población general. En este grupo predomina el TT contuso accidental y por caídas. Al momento de evaluar la gravedad, resulta importante considerar factores propios del paciente como la fragilidad y comorbilidades asociadas, además de los IGT. Creemos que el envejecimiento de la población continuará aumentando la proporción de AM con TT en los próximos años, de ahí lo importante de conocer las características del TT en este grupo etario.

\section{Responsabilidades éticas}

Protección de personas y animales. Los autores declaran que para esta investigación no se han realizado experimentos en seres humanos ni en animales.

Confidencialidad de los datos. Los autores declaran que en este artículo no aparecen datos de pacientes.

Conflictos de interés: no hay.

\section{Bibliografía}

1. Informe mundial sobre el envejecimiento y salud. En: Organización Mundial de la Salud. 2015. WHO/FWC/ALC/15.01.

2. Adulto mayor en Chile. En: Instituto Nacional de Estadísticas. 2017.

3. Urzúa A, Navarrete M. Calidad de vida en adultos mayores: Análisis factoriales de las versiones abreviadas del WHOQoLOld en población chilena. Rev Med Chile 2013;141:28-33.

4. Cares V, Domínguez C, Fernández J, Farías R, Chang W, Fasce G, et al. Evolución de la capacidad funcional en adultos mayores hospitalizados en la Unidad de Geriátrica de Agudos del Hospital Clínico de la Universidad de Chile. Rev Med Chile 2013;141;419-27.

5. Carrasco M, Martínez G, Foradori A, Hoyl T, Valenzuela E, Quiroga T, et al. Identificación y caracterización del adulto mayor saludable. Rev Med Chile 2010;138;1077-83.

6. Magaña I. Trauma en el anciano. Bases anatómicas y fisiológicas para una adecuada evaluación y manejo inicial. Cir Gen. 2007;29:145-8.

7. González R, Riquelme A, Fuentes A, Saldías R, Reyes R, Seguel E, et al. Traumatismo torácico: caracterización de hospitalizaciones durante tres décadas. Rev Med Chile 2018;146:149-58.

8. Aschkenasy M, Rothenhaus T. Trauma and Falls in the Elderly. Emerg Med Clin N Am. 2006;24:413-32.

9. Baker SP, O’Neill B, Haddon W, Long W. The Injury Severity Score: a method for describing patients with multiple injuries and evaluating emergency care. J Trauma 1974; 14:187-96.

10. Champion H, Sacco W, Copes W, Gann D, Gennarelli T, Flanagan M. A revision of the Trauma Score. J Trauma 1989;29:6239.

11. Boyd C, Tolson M, Copes W. Evaluating trauma care: the TRISS method. Trauma Score and Injury Severity Score. J Trauma 1987;27:370-8.

12. Freixinet J, Beltrán J, Rodríguez P, Juliá G, Hussein M, Gil R, et al. Indicators of Severity in Chest Trauma. Arch Bronconeumol. 2008:44:257-62.

13. Mica L, Rufibach K, Keel M, Trentz O. The risk of early mortality of polytrauma patients associated to ISS, NISS, APACHE II values and prothrombin time. J Trauma Manag Outcomes 2013;7:6. Published online 2013 May 24. doi: 10.1186/1752-2897-7-6.

14. Keel M, Trentz O. Pathophysiology of polytrauma. Injury 2005;36:691-9.
15. Ávila R, Hernández A, Marrón C, Hermoso F, Martínez I, Mariscal A, et al. Evolución y complicaciones del traumatismo torácico. Arch Bronconeumol. 2013;49:177-80.

16. Grund S, Roos M, Duchene W, Schuler $M$. Treatment in a center for geriatric traumatology. Dtsch Arztebl Int. 2015;112:113-9.

17. Duvakk D, Zhu X, Elliott A, Wolf S, Rhodes R, Paulk E, et al. Injury severity and comorbidities alone do not predict futility of care after geriatric trauma. J Patiat Med. 2015;18:246-50.

18. Goodmanson N, Rosengart M, Bernato A, Sperry J, Peitzman A, Marshall G. Defining geriatric trauma: When does age make a difference? Surgery 2012;152:66875.

19. Demirhan R, Onan B, Oz K, Halazeroglu S. Comprehensive analysis of 4205 patients with chest trauma: a 10 -year experience. Interact Cardiovasc Thorac Surg. 2009;9:450-3.

20. Dongel I, Coskun A, Ozbay S, Bayram M, Atil B. Management of thoracic trauma in emergency service: Analysis of 1139 cases. Pak J Med Sci. 2013;29:58-63.

21. Al-Koudmani I, Darwish B, Al-Kateb $\mathrm{K}$, Taifour Y. Chest trauma experience 


\section{ARTÍCULO ORIGINAL}

over eleven-year period at al-mouassat university teaching hospital-damascus: a restrospective review of 888 cases. J Cardiothorac Surg. 2012;7:35-42.

22. Kulshrestha P, Munshi I, Wait R. Profile of chest trauma in a level I trauma center. $\mathrm{J}$ Trauma 2004;57:576:81.

23. González R, Riquelme A, Toloza C, Reyes R, Seguel E, Stockins A, et al.
Traumatismo torácico contuso. Rev Chil Enferm Respir 2019; 35: 96-103.

24. González R, Riquelme A, Fuentes A, Canales J, Seguel E, Stockins A, et al. Traumatismo penetrante cardiaco: caracterización, resultados inmediatos y variables asociadas a mortalidad y mortalidad en pacientes operados. Rev Cir. 2019;71:245-52.
25. Lansink KW, Gunning AC, Leenen LP. Cause of death and time of death distribution of trauma patients in a level I trauma center in the Netherlands. Eur J Trauma Emerg Surg. 2013;39:375-83.

26. González R, Riquelme A, Toloza C, Reyes R, Seguel E, Stockins A, et al. Traumatismo torácico por arma blanca. Rev Cir. 2020;72:137-43. 